BIOBIOPROYECTA and its action in the BioBío Region

\section{| RESUMEN |}

La organización nace posterior al terremoto, nuestra principal misión es aportar a la reconstrucción de la Región del Biobío fomentando la participación ciudadana, con un equipo de voluntarios que trabaja activamente en el desarrollo de proyectos aportando con sus conocimientos en el área de la construcción arquitectura y urbanismo.

\section{| ABSTRACT |}

Our oroanization was created after the

earthquake. Our main goal is to contribute to the reconstruction of the Biobío Region promoting citizen participation with a team of volunteers, who actively work in the development of projects and who contribute, with their expertise, in the area of construction, architecture and urbanism.

\title{
BIOBIOPROYECTA y su acción en la Región del Biobío
}

\section{NACE EL VOLUNTARIADO}

Tras la magnitud e impacto del terremoto, surgió una red de voluntarios a nivel nacional que trabajó en las labores de catastros de las viviendas y edificios damnificados tras el terremoto. En nuestra zona, y gracias al apoyo de la página web de reconstruye.cl, grupo que partió como un voluntariado de catastros por profesionales, profesores y estudiantes principalmente de la Pontificia Universidad Católica de Chile y que hoy es una ONG por la producción sustentable del entorno construido, se nos habilitó un espacio de inscripción de voluntarios para la región del Biobío. Esta página fue difundida inicialmente por los medios de comunicación y luego contó con el apoyo del Colegio de Arquitectos de la región, Fosis y Asociación de Municipios, con lo cual se comenzó a formar un equipo de voluntarios del área de la construcción y diseño, los cuales luego de una capacitación masiva realizada en la Universidad de Concepción, comenzaron los trabajos de catastro en la región del Biobío.

El equipo de voluntarios que trabajó en la región, superó las 400 personas, reuniendo a estudiantes y profesionales. La ayuda comenzó a llegar de todos lados; de especial mención es que con mucho entusiasmo recibimos un grupo de voluntarios, estudiantes de la carrera de Arquitectura de la Universidad Austral, quienes apoyaron las labores de catastro desarrolladas en la comuna de Arauco. Nuestros voluntarios estuvieron presentes en las comunas de Talcahuano, San Pedro de la Paz, Lota, Penco, Santa Juana, Florida, Hualqui, Tirúa, Lebu, Los Álamos, Arauco, Cañete, Hualpén, Coelemu, Trehuaco y Larequete. Un trabajo que nos dejó redes sociales creadas y el convencimiento de que nuestra labor como voluntarios no podía quedar sólo ahí. La emergencia la habíamos afrontado con la elaboración de los catastros, posterior a eso, había que comenzar la etapa de la reconstrucción, no quisimos estar ausentes, pues nos sentíamos con el deber y las ganas de aportar con nuestros conocimientos a la reconstrucción de nuestra región.

\section{FUNDACIÓN DE BIOBIOPROYECTA}

Luego de las visitas a las distintas comunas de nuestra región y haber observado en terreno la destrucción que dejó el paso del terremoto en nuestra zona, surge la iniciativa de seguir trabajando. Es entonces como en posteriores reuniones realizadas con los voluntarios que trabajaron en los catastros, se conformó el grupo de acción social denominado BioBiopRoyeCtA (BBP), desde el día 10 de junio del año 2010, dentro del registro de organizaciones comunitarias de la Municipalidad de Concepción, en donde firmaron 18 socios. 
1. Render vista principal "Centro Cívico-Social, Florida». Fuente: A. Cea, A. Acuña, M. Yaconi.

El equipo está compuesto por estudiantes de las carreras de arquitectura e ingeniería y también por profesores de las Universidades de Concepción y San Sebastián. Cabe señalar que BBP nace físicamente en la Universidad de Concepción, institución que tuvo la buena disposición a prestar parte de su infraestructura como apoyo a esta iniciativa de los estudiantes desde el comienzo de los catastros.

Nuestra misión como BBP apunta a servir a la comunidad de la región del Biobío en el proceso de la reconstrucción, y se enfoca en dar apoyo en relación al diseño, construcción, urbanismo y fomentando la participación ciudadana. Para la materialización del proceso nos organizamos en distintas áreas de trabajo, las cuales están encargadas de generar propuestas y actividades según los requerimientos de nuestra región. Así, la organización fue dividida en cuatro líneas de acción las cuales son:

- Catastros: etapa que se desarrolló posterior a la emergencia con más de 400 voluntarios que trabajaron en distintas comunas de la región.

- Asesoría técnica: actividades de orientación en áreas de urbanización, mejoramiento y autoconstrucción para las viviendas de emergencia.

- Gestión y proyectos: elaboración de proyectos de arquitectura y diseño para su posterior postulación a fondos de reconstrucción.

- Investigación y desarrollo: investigación y publicaciones referentes a la reconstrucción y su disponibilidad en portales web.

\section{COMIENZAN LOS PROYECTOS}

Con las redes sociales creadas en las distintas comunas comenzaron a surgir los primeros requerimientos para BBP. Los proyectos realizados se describen a continuación:

\section{CENTRO CÍVICO-SOCIAL, FLORIDA}

Nuestro primer encargo, como BBP, fue el diseño de un «Centro Cívico-Social» para la

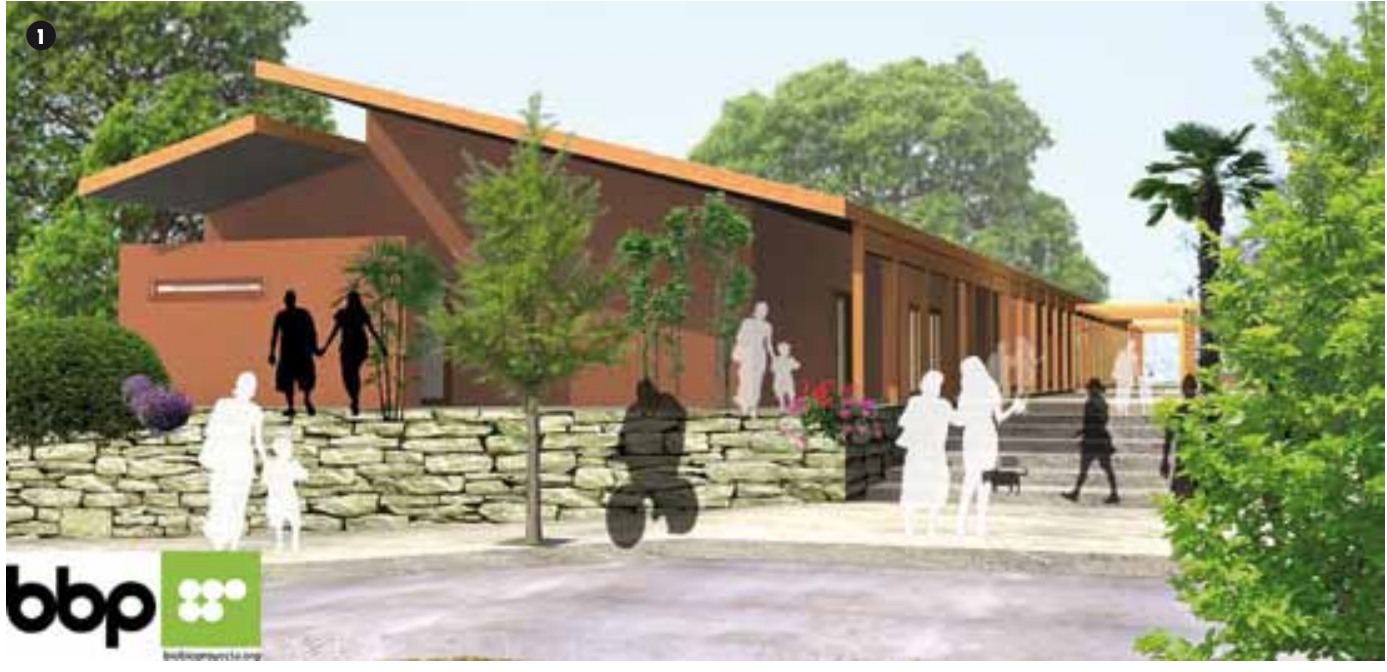

comuna de Florida, trabajo que se gestionó en conjunto con la Municipalidad y un equipo de voluntarios de BBP, quienes viajaron a la comuna y desarrollaron un proyecto de arquitectura, el cual fue postulado a unos fondos relacionados con la reconstrucción (PMU) y que prontamente se construirá en la ciudad.

\section{LOTEO CAMPAMENTO NUEVO AMANECER, DICHATO}

Por otra parte, un equipo de voluntarios fue invitado a visitar la localidad de Dichato, perteneciente a la comuna de Tomé, específicamente el campamento «Nuevo Amanecer», en donde los damnificados por el terremoto se estaban organizando para poder levantar las medias aguas en un terreno alejado del mar. La idea fue recurrir a un equipo de voluntarios, quienes desarrollaron el proyecto de loteo del campamento, el que no llegó a concluirse, pues las autoridades decidieron realizar el diseño del Serviu.

\section{COCINAS RURALES}

En conjunto con AmDel (Asociación de Municipalidades para el Desarrollo Económico Local) se desarrolló el proyecto denominado "Cocinas Rurales», en donde un equipo de voluntarios trabajó en el diseño de nuevas cocinas para las familias damnificadas por el terremoto. El proyecto actualmente se encuentra en busca de financiamiento para poder concretarse.

\section{PROYECTA MEMORIA}

El equipo de Proyectos Urbanos, a mediados de marzo de 2010, crea un proyecto cuyas principales líneas de acción son el reciclaje, patrimonio y espacio público, titulado «Proyecta Memoria». Este proyecto tiene como principales objetivos el poder aportar a resolver dos falencias culturales que poseemos los chilenos, que son la amnesia sísmica y escasez de patrimonio arquitectónico en Chile, a través del reciclaje de los escombros patrimoniales en el espacio público.

En abril de 2010 se desarrolla la "Guía para el reciclaje y recuperación de los escombros simbólicos en el espacio público», el cual fue auspiciado por la Universidad de Concepción, además de ser patrocinada por el Colegio de Arquitectos, Consejo Monumentos Nacionales y CONAma.

Este proyecto tuvo el honor de representar a Chile dentro de los 17 proyectos seleccionados en la $12^{\text {da }}$ Bienal de Venecia 2010 y Bienal de Chile del mismo año, además de adjudicarse un Fondo Nacional para el Fomento del libro y FONDART regional 2011

Dentro de las metas más anheladas de «Proyecta Memoria» es poder consolidar esta nueva postura de reconstrucción patrimonial mundial, 
2. "Cocinas Rurales». Fuente: F. Sánchez, K. Millares, C. Merino, J. Azócar y C. Pérez.

3. "Cocinas Rurales». Fuente: F. Sánchez, K. Millares, C. Merino, J. Azócar y C. Pérez.

4. "Guía para el reciclaje y recuperación de los escombros simbólicos en el espacios públicos. Proyecto Memoria». Fuente: P. Mora, H. Basoalto, V. Vilches, C. Cerda y L. Pérez.

5. "Guía para el Mejoramiento Térmico de la Media Agua». Fuente: C. Nova, E. Landero y M. Flores.

2

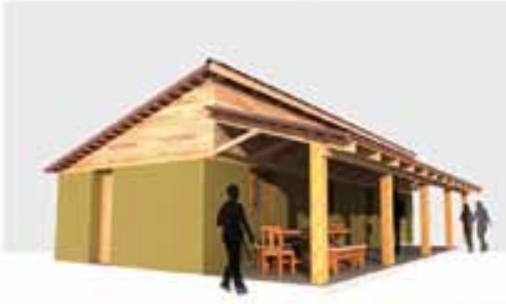

.

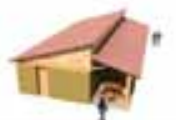

(i)
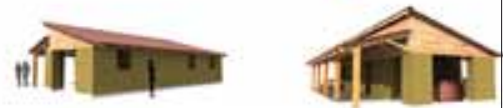

bop

(⿻

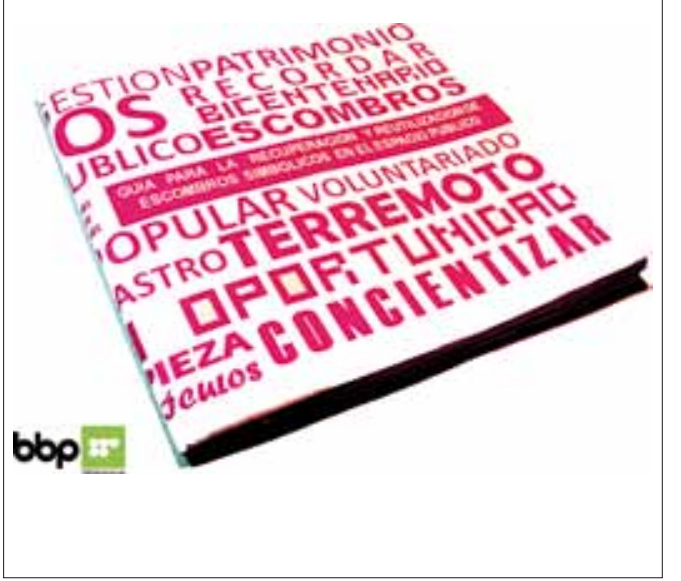

cambiando el concepto de que el patrimonio es un recurso no renovable.

\section{CATASTRO VIVIENDAS PATRIMONIAL RERE}

El municipio de Yumbel solicitó la ayuda de BBP para el catastro de las viviendas patrimoniales de la localidad de Rere. Un equipo de voluntarios en conjunto con Amanda Rivera (arquitecto UBB) realizó la evaluación de daños de la zona de conservación histórica de Rere, elaborando un informe final consistente en fichas de catastros. Con este material el municipio pudo postular a fondos de reparación para los edificios de conservación histórica afectados por el terremoto.

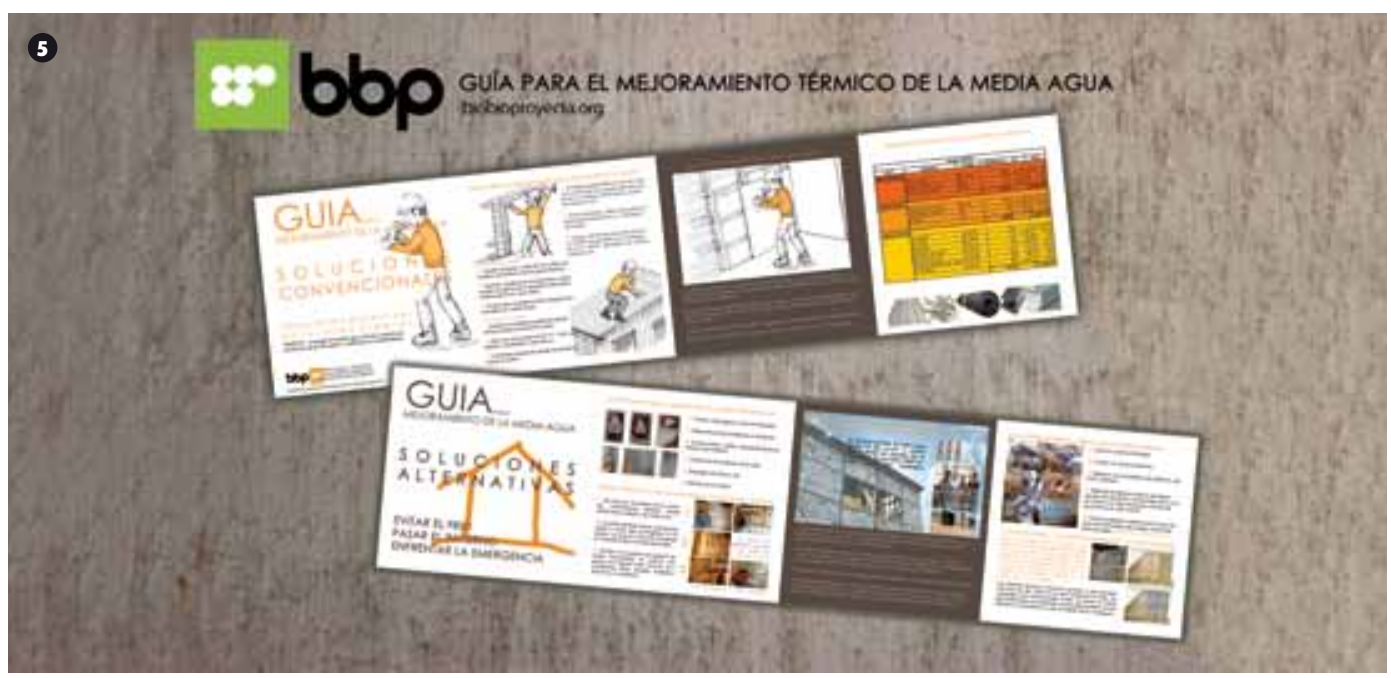

MANUAL MEJORAMIENTO Y AMPLIACIÓN DE MEDIAGUAS

Se elaboró un «Manual para mejoramiento y ampliación de la media agua», trabajo que fue postulado a los fondos de Red Sinergia. Nuestro proyecto no fue ganador, sin embargo, como las redes sociales ya estaban creadas, el manual se hizo llegar a todos nuestros contactos, quienes distribuyeron este texto de orientación entre las familias damnificadas en la región.

\section{ORGANIZACIÓN SEMINARIO «RECONSTRUCCIÓN Y PARTICIPACIÓN, OPORTUNIDADES Y DESAFÍOS} POSTERREMOTO»

El día 19 de octubre el equipo de BBP en conjunto con la FEC (Federación de Estudiantes de la Universidad de Concepción) se organizó el seminario «Reconstrucción y participación, oportunidades y desafíos posterremoto». La idea era poder establecer un diálogo entre la institucionalidad y la ciudadanía, con respecto a los procesos participativos y el proceso de gestión para la implementación de los instrumentos de regulación urbana. Con mucho orgullo podemos manifestar la alta audiencia que tuvo nuestro seminario y la mesa de diálogo que se formó finalmente. 
6. Afiche Seminario «Reconstrucción y participación, oportunidades y desafíos posterremoto». Fuente: P. Mora

7. «Proyecto Memorial Puente Viejo».

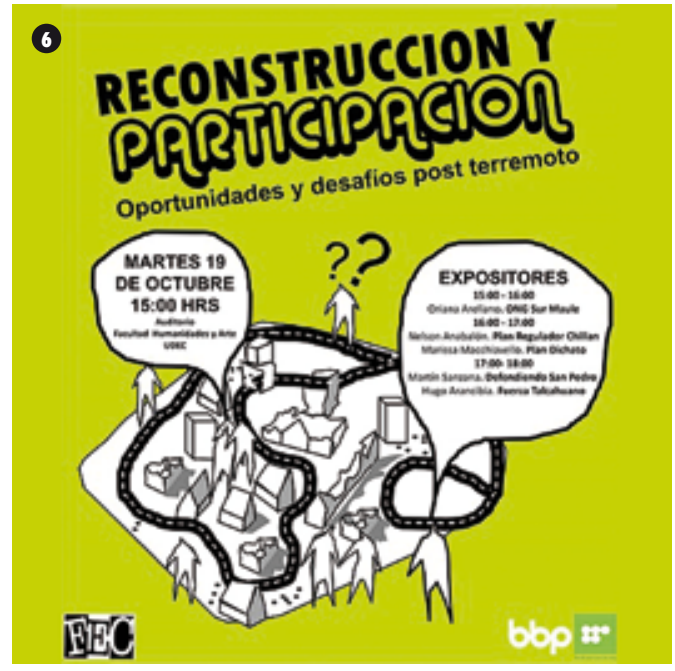

PARTICIPACIÓN EN CONGRESO DE RESPONSABILIDAD SOCIAL UNIVERSITARIA

Fuimos invitados a participar del primer congreso de Responsabilidad Social

Universitaria, desarrollado por la Universidad de la Frontera en Temuco, lugar donde expusimos nuestra experiencia como BBP y conocimos la experiencia de otros grupos de trabajo de acción social.

PUENTE VIEJO. PROYECTO MEMORIAL, RECICLAJE DE ESCOMBROS SIMBÓLICOS

La madrugada del 27 de febrero a las 3:34, un gran terremoto afectó la zona centro-sur de Chile, marcando un punto de inflexión en nuestras vidas y haciéndonos reflexionar sobre nuestras ciudades y su identidad. Vivimos en un país sísmico y tenemos un pasado plasmado en construcciones que han ido desapareciendo, edificios que han sido importantes testigos de nuestra historia y forman parte de nuestra memoria.

Es por esto que detectamos la necesidad de generar intervenciones memoriales donde el patrimonio destruido se reutilice, plasmando recuerdo e identidad en el espacio público, lugar común de nuestro diario vivir. De esta manera se realiza un acto simbólico de «santa sepultura» a estos restos que han marcado por años las vidas chilenas, por medio de proyectos de mejoramiento o creación de nuevos espacios públicos, que pasan a transformarse en entes
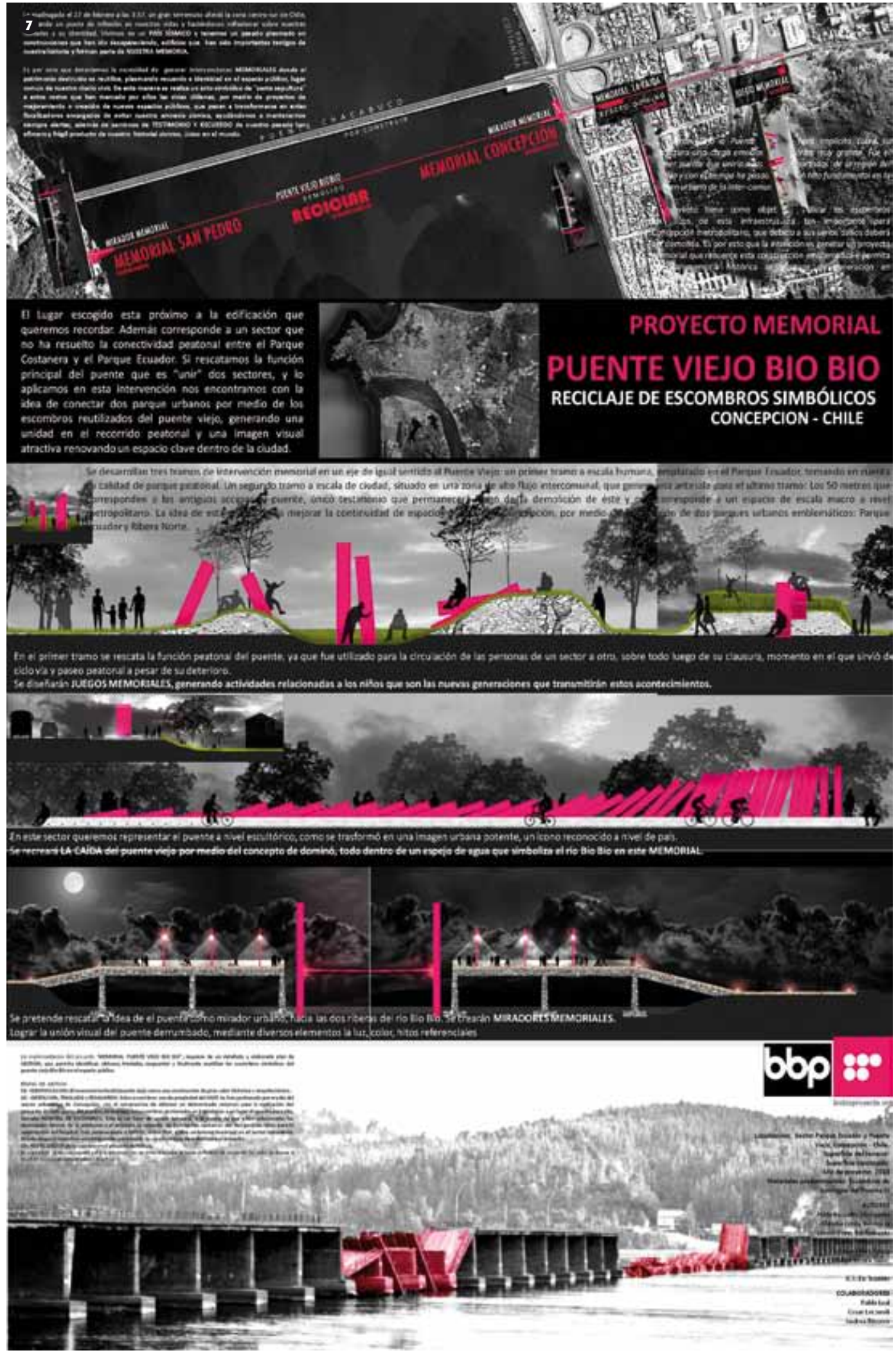


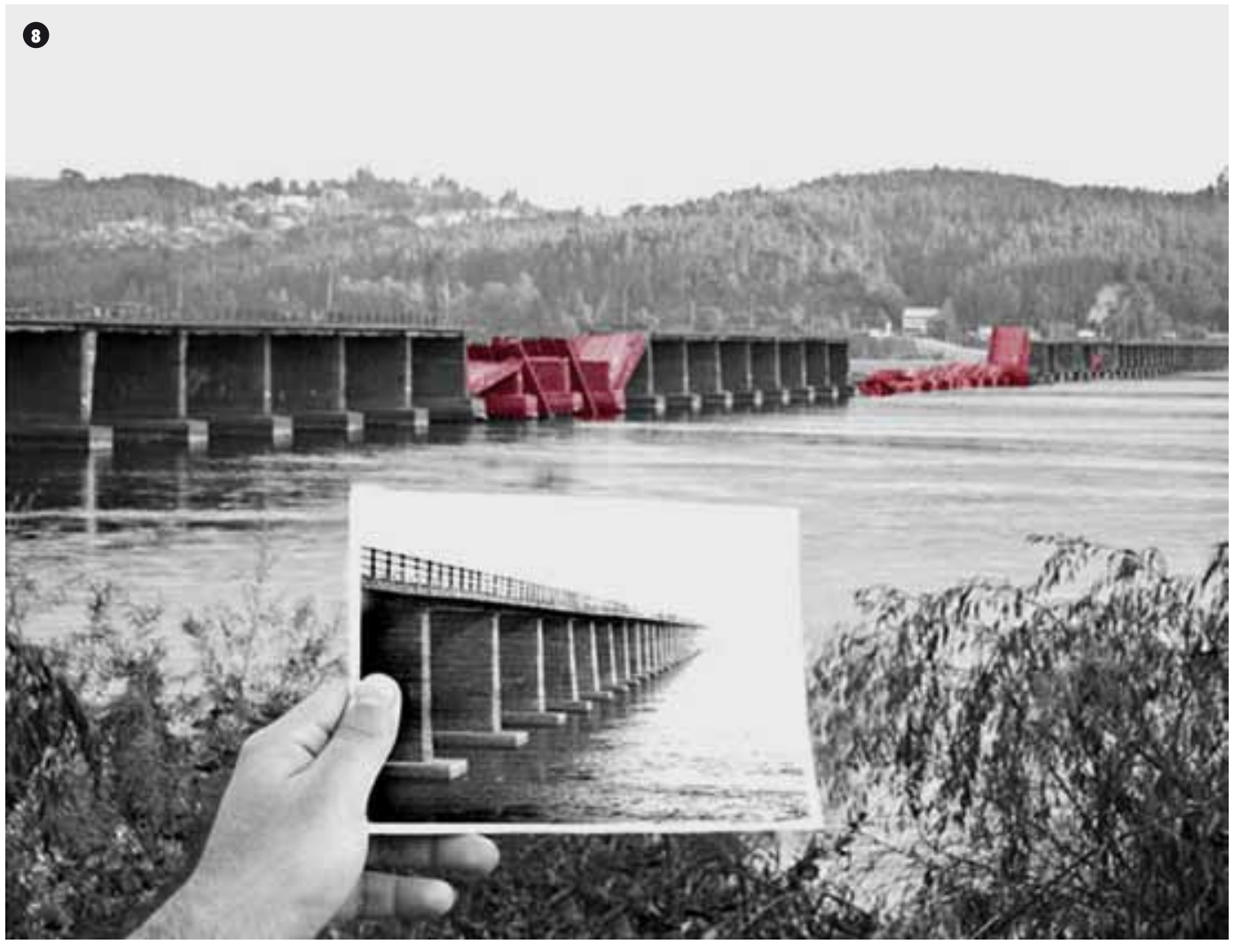

fiscalizadores encargados de evitar nuestra amnesia sísmica, ayudándonos a mantenernos siempre alertas, además de servirnos de testimonio y recuerdo de nuestro pasado tan efímero y frágil producto de nuestro historial sísmico, único en el mundo. El Puente Viejo o puente Biobío, lleva implícito sobre su estructura una carga emocional e histórica muy grande. Fue el primer puente que uniría a las tierras apartadas de la región del Biobío y con el tiempo ha pasado a ser un hito fundamental en la imagen urbana de la intercomuna ribereña.

El proyecto tiene como objetivo reutilizar los escombros simbólicos de esta infraestructura tan importante para Concepción metropolitano, que debido a sus serios daños deberá ser demolida. Es por esto que la intención es generar un proyecto memorial que recuerde esta construcción emblemática y permita que la memoria histórica se traspase de generación en generación.

El lugar escogido está próximo a la edificación que queremos recordar. Además corresponde a un sector que no ha resuelto la conectividad peatonal entre el Parque Costanera y el Parque Ecuador. Si rescatamos la función principal del puente que es «unir» dos sectores, y lo aplicamos en esta intervención nos encontramos con la idea de conectar dos parques urbanos por medio de los escombros reutilizados del puente viejo, generando una unidad en el recorrido peatonal y una imagen visual atractiva renovando un espacio clave dentro de la ciudad.

Se desarrollan tres tramos de intervención memorial en un eje de igual sentido al Puente Viejo: un primer tramo a escala humana, emplazado en el Parque Ecuador, tomando en cuenta su calidad de parque peatonal. Un segundo tramo a escala de ciudad, situado en una zona de alto flujo intercomunal, que genera una antesala para el último tramo: los 50 metros que corresponden a los antiguos accesos al puente, único testimonio que permanecerá luego de la demolición de éste y que corresponde a un espacio de escala macro a nivel metropolitano. La idea de esta secuencia es mejorar la continuidad de espacios públicos de Concepción, por medio de la conexión de dos parques urbanos emblemáticos: Parque Ecuador y Ribera Norte.

En el primer tramo se rescata la función peatonal del puente, ya que fue utilizado para la circulación de las personas de un sector a otro, sobre todo luego de su clausura, momento en el que sirvió de ciclo vía y paseo peatonal a pesar de su deterioro. Se diseñarán juegos memoriales, generando actividades relacionadas a los niños que son las nuevas generaciones que transmitirán estos acontecimientos.

En este sector queremos representar el puente a nivel escultórico, como se trasformó en una imagen urbana potente, un ícono reconocido a nivel de país. Se recreará la caída del puente viejo por medio del concepto de dominó, todo dentro de un espejo de agua que simboliza el río Biobío en este memorial.

Se pretende rescatar la idea del puente como mirador urbano, hacia las dos riberas del río Biobío. Se crearán miradores memoriales. Se pretende lograr la unión visual del puente derrumbado, mediante diversos elementos la luz, color, hitos referenciales.

La implementación del proyecto «Memorial Puente Viejo Biobío», requiere de un detallado y elaborado plan de gestión, que permita identificar, obtener, trasladar, resguardar y finalmente reutilizar los escombros simbólicos del Puente Viejo Biobío en el espacio público.

\section{Etapas de Gestión Proyecto Puente Viejo}

Identificación: El reconocimiento del Puente Viejo como una construcción de gran valor histórico y arquitectónico.

Obtención, traslado y resguardo: Estos escombros son de propiedad del Mop. Se han gestionado por medio del asesor urbanístico de Concepción, con el compromiso de obtener un determinado volumen para la realización del proyecto. En este punto del proceso de reciclaje, los escombros gestionados se trasladarán a un lugar dispuesto para ello, llamado hospital de escombros. Éste es un lugar de acopio temporal, a la espera de que estén solucionadas las necesidades básicas de la población y el proyecto se concrete. En Concepción contamos 
con dos posibles sitios para la construcción del hospital: uno perteneciente a SERVIU, sector Prat, y otro un terreno municipal en el sector cementerio. En esta etapa el escombro será intervenido, para lograr las características deseables para el proyecto.

Reutilización del escombro en el proyecto definitivo: El escombro ya acondicionado para la intervención, se debe trasladar al lugar definitivo de proyecto. Se debe gestionar a nivel de municipalidad el traslado de éstos.

Ficha técnica:

- Localización: sector Parque Ecuador y Puente Viejo, Concepción, Chile.

- Año de proyecto: 2010.

- Materiales predominantes: escombros de hormigón del puente Viejo.

- Autores: Hilda Basoalto Mosquera, Claudia Cerda Inostroza, Leonel Pérez Bustamante, Viviana Vilches Wolf, Patricio Mora Araya y Felipe Rivera Yáñez.

- Colaboradores: Pablo Leal, César Lerzundi y Andrea Rioseco.

\section{DESAFÍOS Y FUTURO DE BBP}

El rumbo de nuestra organización en la actualidad, luego de todos los proyectos realizados con el equipo de voluntarios, ha creado una misión más significativa dentro de la organización: el valor que tomaría la reconstrucción, pero con la participación activa de los ciudadanos. Es así como tenemos en mente un proyecto para fortalecer y potenciar a la sociedad civil a través de un manual denominado «Mapa Vivo Conce», cuya idea es generar lazos entre las organizaciones sociales de Concepción existentes, a través de espacios de encuentro de similares fines que develen lugares importantes para éstos en la ciudad e invitarlos a recorrerlos, como por ejemplo lugares de patrimonio, puntos de reciclaje, centros sociales y culturales. Éste

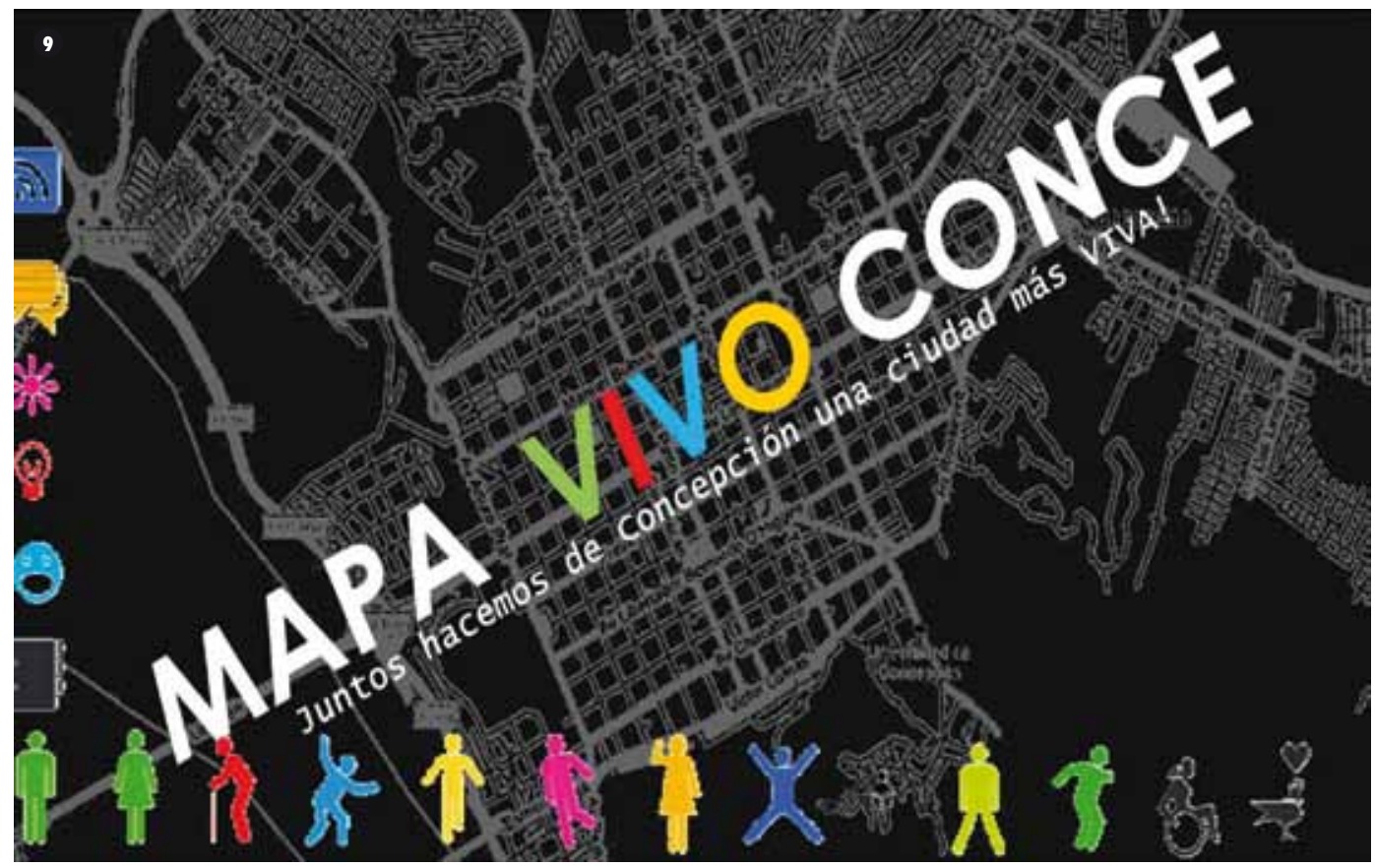

será un manual que ayudará a vivir mejor la ciudad, mostrando múltiples alternativas para hacer un uso más sustentable de Concepción y descubrir dónde el ciudadano puede nutrir sus ganas de participar. Será una herramienta de educación ciudadana desde la comunidad, para la comunidad.

Este nuevo rumbo de BBp ha fortalecido aún más nuestra organización, generando nuestra existencia como miembros en otros proyectos tales como los Programas de Cooperación

Exterior, pertenecientes a la Oficina de Cooperación EuropeAid de la Comisión Europea; trabajando activamente en una red de organizaciones de apoyo tras el terremoto que se desarrolló en la ciudad denominado «Redconstruyamos», en donde a través de una feria participamos con un stand exponiendo nuestra experiencia como grupo de acción social posterremoto.
Actualmente estamos organizando en conjunto con el Centro Cultural Comunitario Pabellón 83, un seminario de participación ciudadana denominado «De la Ciudad Minera a una Nueva Lota» Oportunidades y Desafíos en la Gestión Urbana de Lota, el cual será convocado por la Municipalidad de Lota y tratará de replantear la antigua ciudad minera, hacia una nueva ciudad que rescate su turismo histórico y las redes entre los distintos actores públicos y privados de la ciudad. El seminario se desarrollará el día 14 de enero de 2011.

Como grupo de acción social queremos dejar abierta la invitación a formar parte de nuestro equipo de voluntarios o apoyar con ideas e iniciativas en el correo biobioproyecta@gmail.com 